\title{
Vital-SCOPE: Design and Evaluation of a Smart Vital Sign Monitor for Simultaneous Measurement of Pulse Rate, Respiratory Rate, and Body Temperature for Patient Monitoring
}

\author{
Guanghao Sun $\mathbb{D},{ }^{1}$ Takemi Matsui $\mathbb{D}^{2},{ }^{2}$ Yasuyuki Watai, ${ }^{2}$ Seokjin Kim, ${ }^{2}$ Tetsuo Kirimoto, ${ }^{1}$ \\ Satoshi Suzuki, ${ }^{3}$ and Yukiya Hakozaki ${ }^{4}$ \\ ${ }^{1}$ Graduate School of Informatics and Engineering, The University of Electro-Communications, Tokyo, Japan \\ ${ }^{2}$ Graduate School of System Design, Tokyo Metropolitan University, Tokyo, Japan \\ ${ }^{3}$ Department of Mechanical Engineering, Kansai University, Osaka, Japan \\ ${ }^{4}$ Genkikai Yokohama Hospital, Yokohama, Japan
}

Correspondence should be addressed to Guanghao Sun; guanghao.sun@uec.ac.jp

Received 5 August 2017; Revised 26 November 2017; Accepted 6 December 2017; Published 1 February 2018

Academic Editor: Armando Ricciardi

Copyright (c) 2018 Guanghao Sun et al. This is an open access article distributed under the Creative Commons Attribution License, which permits unrestricted use, distribution, and reproduction in any medium, provided the original work is properly cited.

\begin{abstract}
Consistent vital sign monitoring is critically important for early detection of clinical deterioration of patients in hospital settings. Mostly, nurses routinely measure and document the primary vital signs of all patients 2-3 times daily to assess their condition. To reduce nurse workload and thereby improve quality of patient care, a smart vital sign monitor named "Vital-SCOPE" for simultaneous measurement of vital signs was developed. Vital-SCOPE consists of multiple sensors, including a reflective photo sensor, thermopile, and medical radar, to be used in simultaneous pulse rate, respiratory rate, and body temperature monitoring within $10 \mathrm{~s}$. It was tested in laboratory and hospital settings. Bland-Altman and Pearson's correlation analyses were used to compare the Vital-SCOPE results to those of reference measurements. The mean difference of the respiratory rate between respiratory effort belt and Vital-SCOPE was 0.47 breaths per minute with the $95 \%$ limit of agreement ranging from -7.4 to 6.5 breaths per minute. The Pearson's correlation coefficient was $0.63(P<0.05)$. Moreover, the mean difference of the pulse rate between electrocardiogram and Vital-SCOPE was 3.4 beats per minute with the 95\% limit of agreement ranging from -13 to 5.8 beats per minute; the Pearson's correlation coefficient was $0.91(P<0.01)$, indicating strong linear relationship.
\end{abstract}

\section{Introduction}

Consistent vital sign monitoring is critically important for early detection of clinical deterioration in hospital settings [1]. In most hospitals, nurses routinely measure and document primary vital signs (e.g., pulse rate, oxygen saturation, respiratory rate, and body temperature) for all patients $2-3$ times per day to make an assessment of the patient's condition $[2,3]$. However, such measurement and documentation is a repetitive and time-consuming task for nurses [4]. They use different medical devices such as an electronic thermometer to measure body temperature and a pulse oximeter to measure heart rate and oxygen saturation. In particular, the measurement of respiratory rate is often omitted because having nurses count the chest wall movement in a busy hospital setting is not efficient $[5,6]$. To reduce the workload of the nurses and thus improve the quality of patient care, we developed a smart vital sign monitor for simultaneous measurement of pulse rate, respiratory rate, and body temperature.

In our previous studies, we proposed a multiple vital sign-based infection screening system with sensor fusion technology that can rapidly perform medical inspections at places of mass gatherings, such as airport quarantine stations 
and outpatient units [7-10]. This multiple sensor system consists of a medical radar and an infrared thermographic camera. Pulse and respiratory rates were measured using a $24 \mathrm{GHz}$ medical radar, and body surface temperature was monitored using a thermographic camera without contacting the patient. We tested the system on patients with seasonal influenza in case-controlled studies and showed that the detection sensitivity ranged from $81.5 \%$ to $98.0 \%$. The advantages of this system include the following: (1) rapid and simultaneous measurement of multiple vital signs within $10 \mathrm{~s}$; (2) a noncontact measurement scheme for reducing the physical and mental burden of patients considerably; and (3) automatic detection of clinical deterioration with optimal discriminant analysis.

These advantages compelled us to redesign the infection screening system to create a smart, portable, and easy-to-use vital sign monitor for patient monitoring. To miniaturize the vital sign monitor, an onboard field programmable gate array (FPGA) microcontroller was used to acquire and process the biosignals from multiple sensors. In addition, we integrated multiple sensors (reflective photo sensor, thermopile, and $24 \mathrm{GHz}$ medical radar) and a FPGA microcontroller into a small disc-shaped form similar to a stethoscope, thus arriving at the name "Vital-SCOPE." Throughout the remainder of this paper, we describe the design concept of Vital-SCOPE and report the validation of this system in both laboratory and hospital settings.

\section{Material and Methods}

2.1. The Design Concept and Hardware Configuration of Vital-SCOPE. Vital-SCOPE simultaneously measures pulse rate, respiratory rate, and body temperature when the sensor unit is placed on the carotid artery area of the neck and provides results within $10 \mathrm{~s}$ (Figure 1). The Vital-SCOPE sensor unit consists of a $24 \mathrm{GHz}$ medical radar (SHARP, DC6M4JN3000, Japan), reflective photo sensor (Pulse Sensor, SEN-11574, SparkFun), and thermopile contactless temperature sensor (NIPPON CERAMIC, TSUP-A1D01S50-60, Japan). The respiratory rate is measured with a $24 \mathrm{GHz}$ medical radar by monitoring the chest surface movements. Note that the output power of the $24 \mathrm{GHz}$ medical radar is $10 \mathrm{~mW}$ with an electric power density of $1.5 \times 10^{-2} \mathrm{~mW} / \mathrm{cm}^{2}$ at the carotid artery area of the neck, which is much lower than the Japanese safety guideline for radio-radiation protection. The reflective photo sensor is used to measure pulse rate from the carotid artery, and the body surface temperature is measured using the thermopile. The disc-shaped sensor unit is $5.5 \mathrm{~cm}$ in diameter and $6.0 \mathrm{~cm}$ in height and can be easily used in one-handed operations.

A block diagram of Vital-SCOPE is illustrated in Figure 2. A myRIO-embedded device from National Instruments [11] is used to control the entire system; myRIO includes analog inputs, a WiFi module, Xilinx FPGA, and a dual-core ARM Cortex-A9 processor. As shown in Figure 2, the body temperature value is the output from the thermopile and is sent to myRIO via I2C communications.
Simultaneously, the respiratory and pulse signals measured by the $24 \mathrm{GHz}$ medical radar and photo sensor are converted to digital signals with a sampling rate of $100 \mathrm{~Hz}$ for data transfer via the IO ports. Moreover, a signal-processing printed circuit board (PCB) was designed to amplify the $24 \mathrm{GHz}$ medical radar signal, as well as a band-pass filter to eliminate DC components and high-frequency noise (set between $0.1 \mathrm{~Hz}$ and $0.7 \mathrm{~Hz}$ for respiration signal). The raw data acquired by the multiple sensors were saved in an external USB flash drive connected to myRIO inside the device. The calculated pulse rate, respiratory rate, and body temperature are sent wirelessly using the WiFi module to a tablet PC in real time, where automated documentations of the vital signs are stored and displayed. The battery consumption is important for designing such a hand-held system. The multiple sensors and the myRIO-embedded device have low current consumption of between 1000 and $1500 \mathrm{~mA}$. In this work, an $8400 \mathrm{mAh}$ mobile battery was used for powering the system, which means that the system lasts for approximately 5.6 hours in continuous use. The myRIO-embedded device and battery are placed in the controller unit. The controller unit is $25.0 \mathrm{~cm}$ in width, $10.5 \mathrm{~cm}$ in depth, and $24.0 \mathrm{~cm}$ in height.

\subsection{Evaluation of Vital-SCOPE in Laboratory and Hospital} Settings. The evaluation of Vital-SCOPE was conducted in both laboratory and hospital settings. Ten graduate students $(22.7 \pm 1.3$ years of age) participated in the laboratory test at Tokyo Metropolitan University. To cover a wide range of pulse and respiratory rates, the test procedure was divided into two sessions. First, the subjects were tested at rest, and second, the subjects were tested just after exercising. The exercise trial consisted of the subjects using a bicycle ergometer at $100 \mathrm{~W}$ for $15 \mathrm{~min}$ after taking the measurement during a resting state, thereby elevating the pulse and respiratory rates. Their respiratory rate, pulse rate, and body surface temperature were measured by Vital-SCOPE; reference measurements were simultaneously obtained using a contacttype electrocardiogram (ECG) (GMS, LRR-03, Japan) and a respiratory effort belt (NIHON KOHDEN, TR-512, Japan). In order to evaluate the viability of Vital-SCOPE in a hospital setting, we tested it with 11 inpatients $(72.2 \pm 15.5$ years of age) at Yokohama Hospital in February 2017 for one week. The Yokohama Hospital is a recuperation hospital for care of older people. This study was approved by the Ethics Committee of the Yokohama Hospital and the Committee on Human Research of the Faculty of System Design, Tokyo Metropolitan University. All subjects gave their informed written consent.

\subsection{Statistical Analysis. The Bland-Altman and Pearson's} correlation analysis were used to compare Vital-SCOPE to the reference measurements obtained using the MATLAB Statistics and Machine Learning Toolbox (MathWorks, Natick, MA, USA). The Bland-Altman analysis is a simple and efficient method to assess the agreement between two measurements in clinical studies [12]. A $P$ value of less than 0.05 is considered to indicate statistical significance. 

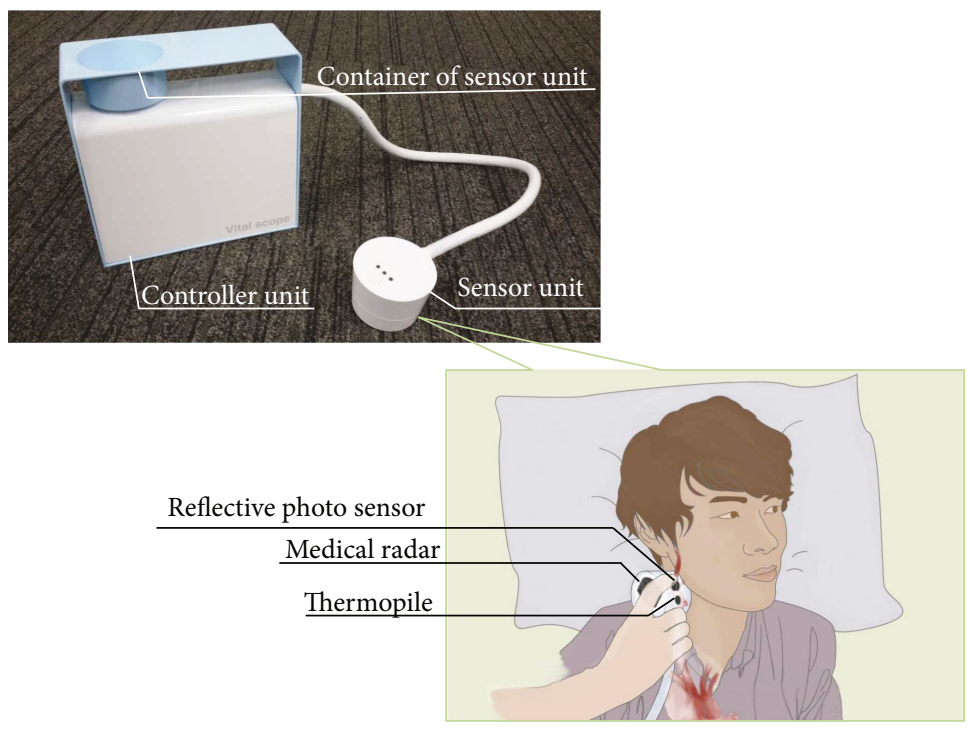

FIGURE 1: Design concept and hardware configuration of Vital-SCOPE.

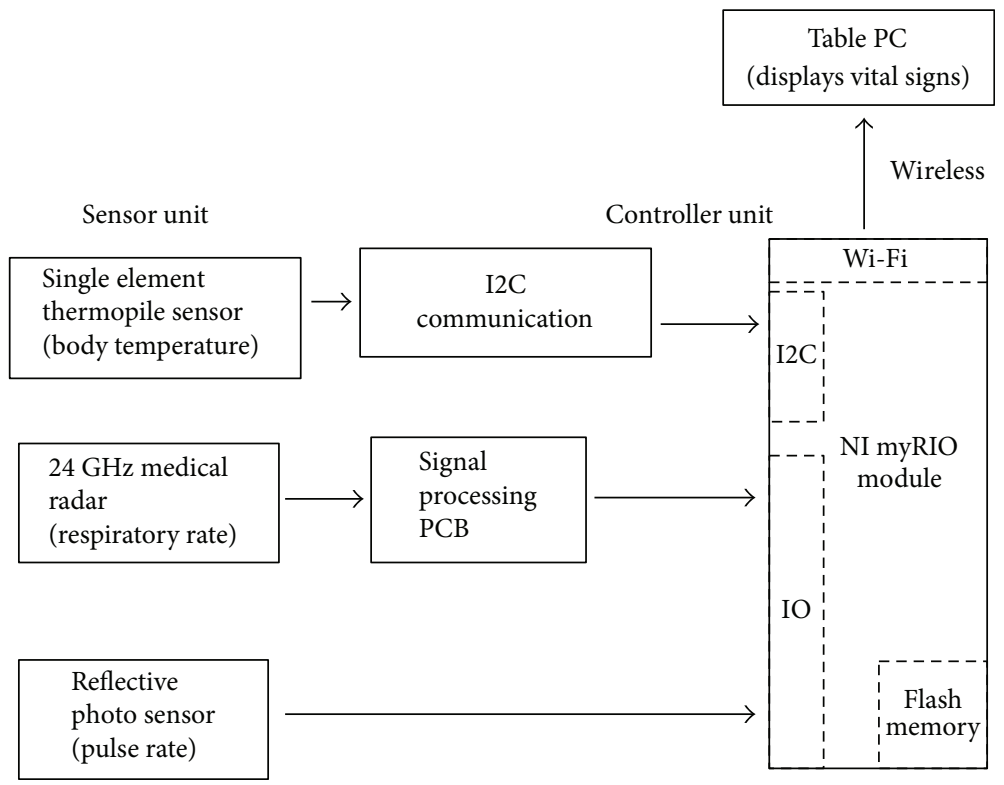

FIGURE 2: Block diagram of Vital-SCOPE.

\section{Results}

Figure 3 shows the Bland-Altman and Pearson's correlation plots for ten participants with 160 pairs of respiratory rate (resting condition only) and 320 pairs of pulse rate (rest and after exercising) measurements in the laboratory. The mean difference of the respiratory rate between the respiratory effort belt and Vital-SCOPE was 0.47 breaths per minute (bpm) with the $95 \%$ limit of agreement ranging from -7.4 to $6.5 \mathrm{bpm}$. The Pearson's correlation coefficient was $0.63(P<0.05)$, indicating a moderately strong linear relationship. Moreover, the mean difference of the pulse rate between the ECG and Vital-SCOPE was 3.4 beats per minute (bpm) with the
95\% limit of agreement ranging from -13 to $5.8 \mathrm{bpm}$; the Pearson's correlation coefficient was $0.91(P<0.01)$ showing a strong linear relationship. Figure 4 shows a comparison of the pulse and respiratory signals measured using VitalSCOPE, ECG, and the respiratory effort belt. The peaks of the signal pulse were similar to the R-R interval in an ECG signal. In addition, the respiratory signal measured using a medical radar within $10 \mathrm{~s}$ indicated a high correlation with the signal measured by the respiratory effort belt.

We also evaluated the usability of Vital-SCOPE in a hospital setting where the nurses checked the vital signs of patients every day for a week. Table 1 presents the historical trends of pulse rate, respiratory rate, and body surface 
Bland-Altman plot of pulse rate
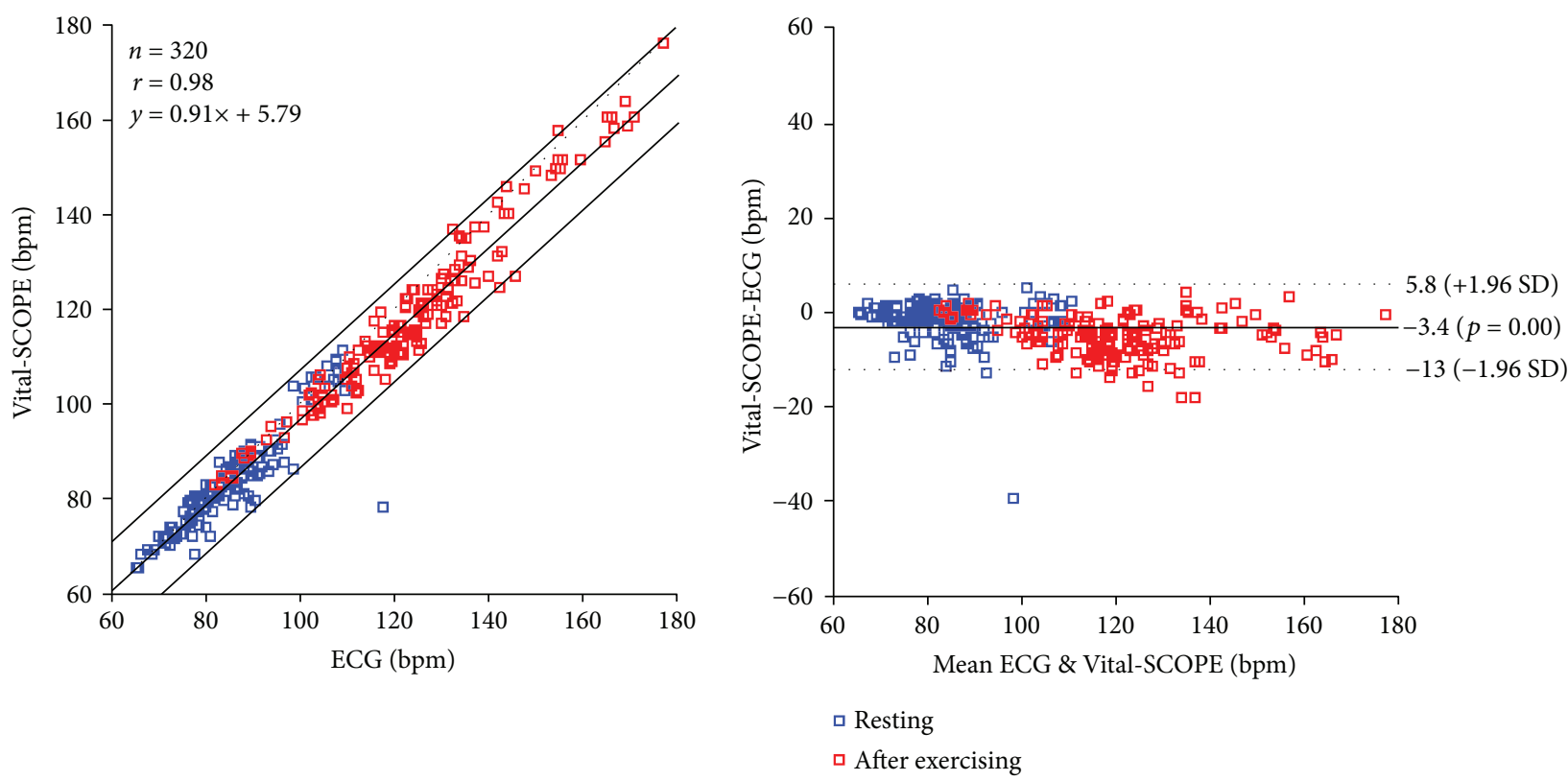

Bland-Altman plot of respiratory rate
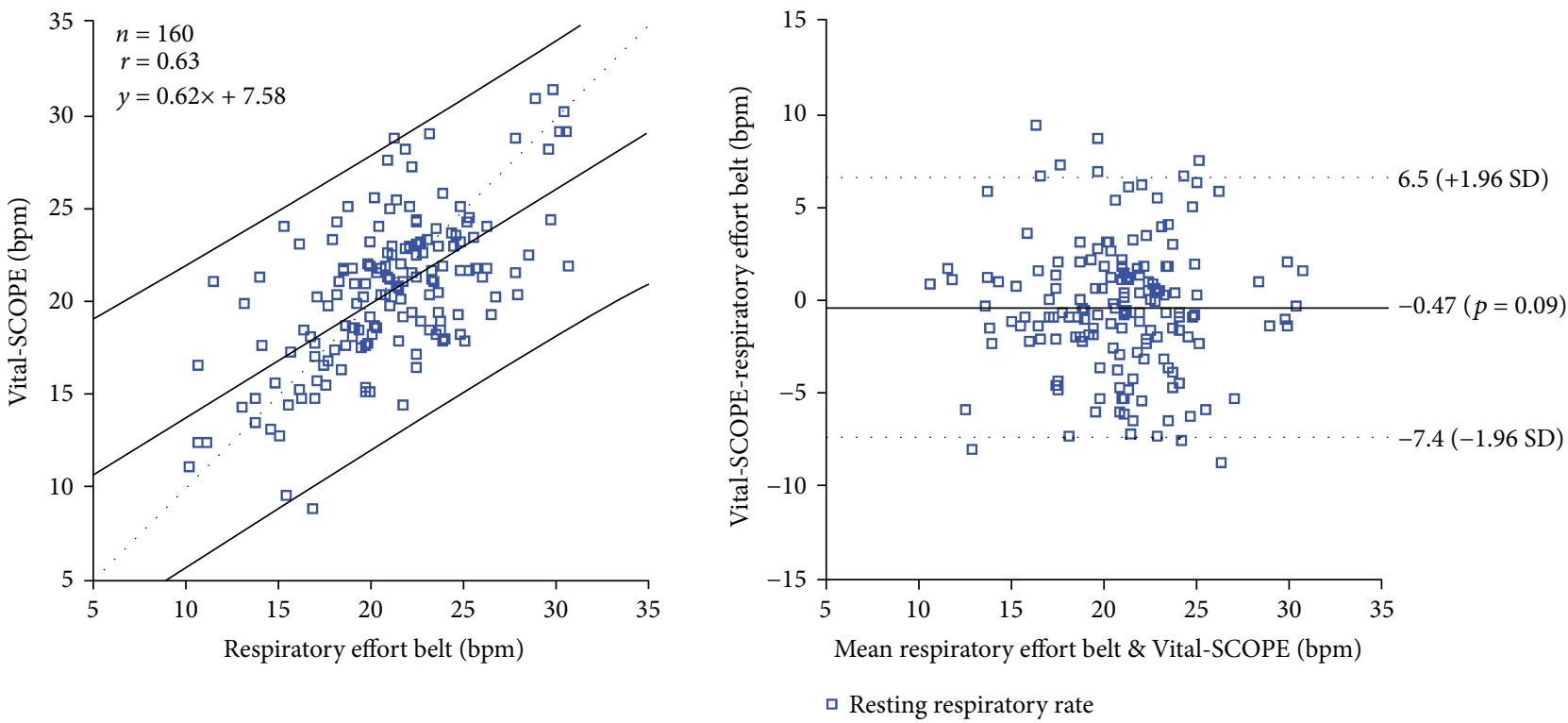

FIgURE 3: Bland-Altman and Pearson's correlation plots of Vital-SCOPE, ECG, and respiratory effort belt.

temperature measured via Vital-SCOPE for four patients at Yokohama Hospital. The comparison of the body surface temperature and axillary temperature showed an average mean difference of $1.5^{\circ} \mathrm{C}$, owing to the body surface temperature being lower than the core temperature [13]. As observed in Table 1, patient B had a fever (axillary temperature: $38.5^{\circ} \mathrm{C}$, body surface temperature: $37.1^{\circ} \mathrm{C}$ ) on February 24,2017 , indicating that Vital-SCOPE accurately captured the elevated body temperature.

\section{Discussion}

In this paper, a vital sign monitor was proposed for patient monitoring that simultaneously measures pulse rate, respiratory rate, and body temperature. We investigated the use of Vital-SCOPE in laboratory and hospital settings and compared the vital sign measurements with reference devices. The results showed that Vital-SCOPE provides the possibility of accurate vital sign measurements that can reduce the 

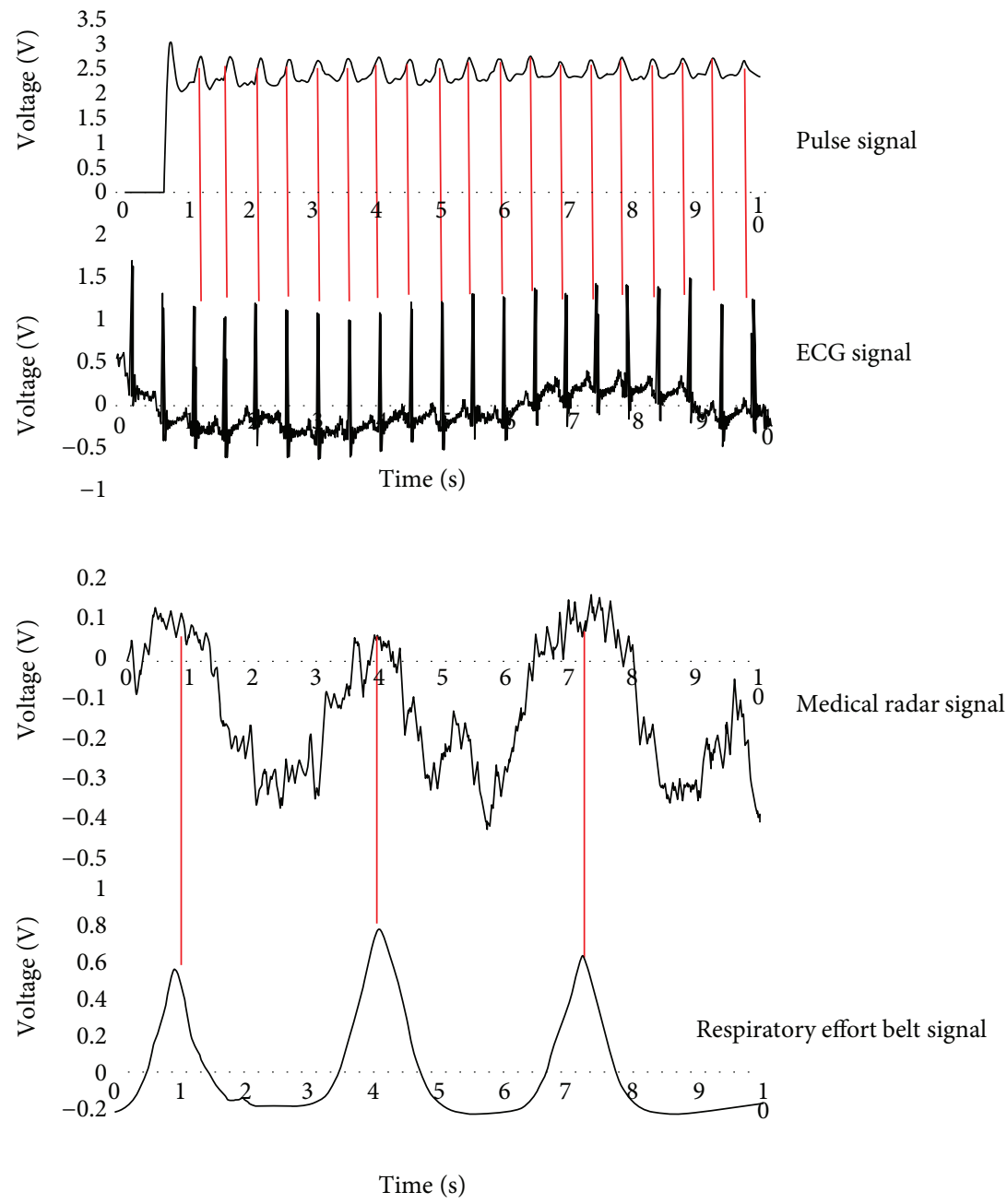

FIGURE 4: A comparison of the pulse and respiratory signals measured via Vital-SCOPE, ECG, and respiratory effort belt.

workload of nurses and improve patient care. Contact-type ECG and respiratory belt transducer have become universally accepted standard for measuring heart and respiratory rates. However, such measurements require the attachment of sensors, which can cause discomfort and place a heavy burden on patient. In recent years, a variety of unobtrusive medical sensors have been developed for long-term or intermittent physiological measurements, including RGB visible and thermal images for measuring heart and respiratory rates [7] and measurement of cardiac and respiration pulses using laser Doppler or a microwave medical radar $[14,15]$. These noncontact sensors have their advantages and disadvantages depending on the specific applications. In this study, we adopted a medical radar combined with a reflective photo sensor and thermopile to measure multiple vital signs; this sensor fusion device offered advantages of vital sign information integration for early detection of clinical deterioration in hospital settings.

To date, body temperature, pulse rate, and oxygen saturation are usually checked by nurses using an electronic thermometer and pulse oximeter. However, the measurement of the respiratory rate is often omitted because it is not efficient in busy hospital settings. Recent studies indicated that a respiratory rate higher than $27 \mathrm{bpm}$ is an important predictor of cardiac arrest for inpatients in hospital settings [16]. To meet this need, we designed Vital-SCOPE not only to measure body temperature and pulse rate but also for noncontact measurement of the respiratory rate using a medical radar. Because a medical radar is a noncontact-type sensor, the measurement significantly reduced the physical and psychological burden on patients. In this study, as shown in Table 1, the pulse rate and body temperature of some patients were near normal. However, their respiratory rate was higher than $27 \mathrm{bpm}$ with irregular respiratory rhythms. In a case like this, Vital-SCOPE, which simultaneously monitors multiple vital signs including respiratory rate, may offer an early identification of patients who are at risk of clinical deterioration. In addition, Vital-SCOPE combines vital sign acquisition and automatic documentation, helping nurses make their work more efficient.

In this study, we showed the design concept and basic architecture of Vital-SCOPE, but there are several potential limitations of our current system. (1) In such embedded system, to reduce the usage of energy, the minimum sampling rate (in this study $100 \mathrm{~Hz}$ ) is required but without 
TABLE 1: The historical trends of vital signs measured via Vital-SCOPE for four patients at a hospital setting.

\begin{tabular}{|c|c|c|c|c|c|c|c|}
\hline & Day & Age & Gender & $\begin{array}{l}\text { Respiration } \\
\text { rate (bpm) }\end{array}$ & $\begin{array}{l}\text { Pulse rate } \\
\text { (bpm) }\end{array}$ & $\begin{array}{c}\text { Body surface } \\
\text { temperature } \\
\left({ }^{\circ} \mathrm{C}\right)\end{array}$ & $\begin{array}{c}\text { Axillary } \\
\text { temperature } \\
\left({ }^{\circ} \mathrm{C}\right) \\
\end{array}$ \\
\hline \multirow{5}{*}{ Patient A } & $2017 / 2 / 21$ & 81 & Male & 27 & 77 & 32.0 & 37.8 \\
\hline & $2017 / 2 / 22$ & 81 & Male & 27 & 73 & 34.4 & 35.2 \\
\hline & $2017 / 2 / 23$ & 81 & Male & 22 & 67 & 34.6 & 35.9 \\
\hline & $2017 / 2 / 24$ & 81 & Male & 16 & 74 & 34.2 & 35 \\
\hline & $2017 / 2 / 25$ & 81 & Male & 20 & 95 & 34.7 & 36.6 \\
\hline \multirow{5}{*}{ Patient B } & $2017 / 2 / 21$ & 85 & Male & 27 & 78 & 35.4 & 37.1 \\
\hline & $2017 / 2 / 22$ & 85 & Male & 23 & 90 & 35.3 & 36.6 \\
\hline & $2017 / 2 / 23$ & 85 & Male & 27 & 62 & 35.0 & 36.9 \\
\hline & $2017 / 2 / 24$ & 85 & Male & 28 & 71 & 37.1 & 38.5 \\
\hline & $2017 / 2 / 25$ & 85 & Male & 23 & 63 & 35.3 & 35.4 \\
\hline \multirow{5}{*}{ Patient C } & $2017 / 2 / 21$ & 92 & Female & 22 & 83 & 36.8 & 37.3 \\
\hline & $2017 / 2 / 22$ & 92 & Female & 26 & 87 & 35.4 & 37.9 \\
\hline & $2017 / 2 / 23$ & 92 & Female & 25 & 67 & 36.1 & 37.2 \\
\hline & $2017 / 2 / 24$ & 92 & Female & 21 & 75 & 36.7 & 36.6 \\
\hline & $2017 / 2 / 25$ & 92 & Female & 28 & 79 & 35.5 & 37.4 \\
\hline \multirow{5}{*}{ Patient D } & $2017 / 2 / 21$ & 68 & Female & 25 & 75 & 34.7 & 35.6 \\
\hline & $2017 / 2 / 22$ & 68 & Female & 13 & 84 & 34.4 & 36.2 \\
\hline & $2017 / 2 / 23$ & 68 & Female & 23 & 91 & 34.6 & 35.8 \\
\hline & $2017 / 2 / 24$ & 68 & Female & 34 & 75 & 33.8 & 35.1 \\
\hline & $2017 / 2 / 25$ & 68 & Female & 32 & 76 & 35.5 & 36 \\
\hline
\end{tabular}

compromising the accuracy. (2) Regarding the automatic documentation of measured vital signs, we adopted the Data Dashboard for LabVIEW app on a tablet PC to visualize the data; but we are still working on designing an easy-to-use graphical user interface for nurses. (3) In this study, we focused on evaluating the measurement accuracy of the system, however without including the function of automatic detection of clinical deterioration. In our near future work, we will include the national early warning score (NEWS) to our system based on measured vital signs. The NEWS score was introduced by the Royal College of Physicians in London; the score parameters include respiratory rate, oxygen saturation level, body temperature, blood pressure, and heart rate. The higher score indicates higher risk and significant clinical deterioration [17]. To this time, our system only measures three parameters of NEWS score; we will add other vital signs such as blood pressure and oxygen saturation in our next work.

\section{Conclusion}

In summary, a multiple vital sign monitor "Vital-SCOPE" was designed for patient monitoring. Because the system simultaneously monitors pulse rate, respiratory rate, and body temperature within $10 \mathrm{~s}$ and automatically documents the vital signs, it has high potential for use as an important tool in patient monitoring in a hospital setting.

\section{Conflicts of Interest}

The authors have no conflict of interest.

\section{Acknowledgments}

This study was supported by a Grant-in-Aid for Young Scientists (16K16363) funded by the Ministry of Education, Culture, Sports, Science and Technology of Japan. The authors are grateful to the nursing staff at Yokohama Hospital for their contribution to this study.

\section{References}

[1] M. Elliott and A. Coventry, "Critical care: the eight vital signs of patient monitoring," British Journal of Nursing, vol. 21, no. 10, pp. 621-625, 2012.

[2] W. Q. Mok, W. Wang, and S. Y. Liaw, "Vital signs monitoring to detect patient deterioration: an integrative literature review," International Journal of Nursing Practice, vol. 21, no. S2, pp. 91-98, 2015.

[3] T. Watkins, L. Whisman, and P. Booker, "Nursing assessment of continuous vital sign surveillance to improve patient safety on the medical/surgical unit," Journal of Clinical Nursing, vol. 25, no. 1-2, pp. 278-281, 2016.

[4] L. Rose and S. P. Clarke, "Vital signs," The American Journal of Nursing, vol. 110, no. 5, p. 11, 2010. 
[5] S. Cooper, R. Cant, and L. Sparkes, "Respiratory rate records: the repeated rate?," Journal of Clinical Nursing, vol. 23, no. 9-10, pp. 1236-1238, 2014.

[6] M. A. Cretikos, R. Bellomo, K. Hillman, J. Chen, S. Finfer, and A. Flabouris, "Respiratory rate: the neglected vital sign," The Medical Journal of Australia, vol. 188, no. 11, pp. 657-659, 2008.

[7] G. Sun, Y. Nakayama, S. Dagdanpurev et al., "Remote sensing of multiple vital signs using a CMOS camera-equipped infrared thermography system and its clinical application in rapidly screening patients with suspected infectious diseases," International Journal of Infectious Diseases, vol. 55, pp. 113-117, 2017.

[8] G. Sun, T. Matsui, Y. Hakozaki, and S. Abe, "An infectious disease/fever screening radar system which stratifies higherrisk patients within ten seconds using a neural network and the fuzzy grouping method," The Journal of Infection, vol. 70, no. 3, pp. 230-236, 2015.

[9] Y. Yao, G. Sun, T. Matsui, Y. Hakozaki, S. van Waasen, and M. Schiek, "Multiple vital-sign-based infection screening outperforms thermography independent of the classification algorithm," IEEE Transactions on Biomedical Engineering, vol. 63, no. 5, pp. 1025-1033, 2016.

[10] T. Matsui, Y. Hakozaki, S. Suzuki et al., "A novel screening method for influenza patients using a newly developed noncontact screening system," Journal of Infection, vol. 60, no. 4, pp. 271-277, 2010.

[11] G. Sun, Y. Watai, and T. Matsui, "Development of a wireless physiological computing platform using National Instruments' myRIO embedded device," in Proceedings of 2015 IEEE 4th Global Conference on Consumer Electronics, pp. 27-30, Osaka, Japan, October 2015.

[12] D. Giavarina, "Understanding Bland Altman analysis," Biochemia Medica, vol. 25, no. 2, pp. 141-151, 2015.

[13] G. Sun, T. Matsui, T. Kirimoto, Y. Yao, and S. Abe, “Applications of infrared thermography for noncontact and noninvasive mass screening of Febrile International Travelers at airport quarantine stations," in Application of Infrared to Biomedical Sciences, Y. K. Ng and M. Etehadtavakol, Eds., pp. 347-358, Springer, Singapore, 2017.

[14] C. Li, V. M. Lubecke, O. Boric-Lubecke, and J. Lin, "A review on recent advances in Doppler radar sensors for noncontact healthcare monitoring," IEEE Transactions on Microwave Theory and Techniques, vol. 61, no. 5, pp. 2046-2060, 2013.

[15] S. Ulyanov and V. Tuchin, "Pulse-wave monitoring by means of focused laser beams scattered by skin surface and membranes," in Proceedings SPIE 1884, Static and Dynamic Light Scattering in Medicine and Biology, pp. 160-168, Los Angeles, CA, 1993.

[16] J. F. Fieselmann, M. S. Hendryx, C. M. Helms, and D. S. Wakefield, "Respiratory rate predicts cardiopulmonary arrest for internal medicine inpatients," Journal of General Internal Medicine, vol. 8, no. 7, pp. 354-360, 1993.

[17] S. Uppanisakorn, R. Bhurayanontachai, J. Boonyarat, and J. Kaewpradit, "National Early Warning Score (NEWS) at ICU discharge can predict early clinical deterioration after ICU transfer," Journal of Critical Care, vol. 43, pp. 225-229, 2017. 


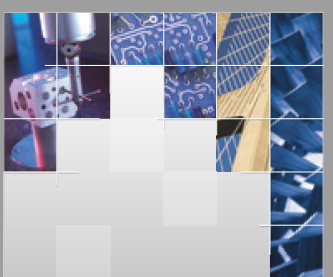

\section{Enfincering}
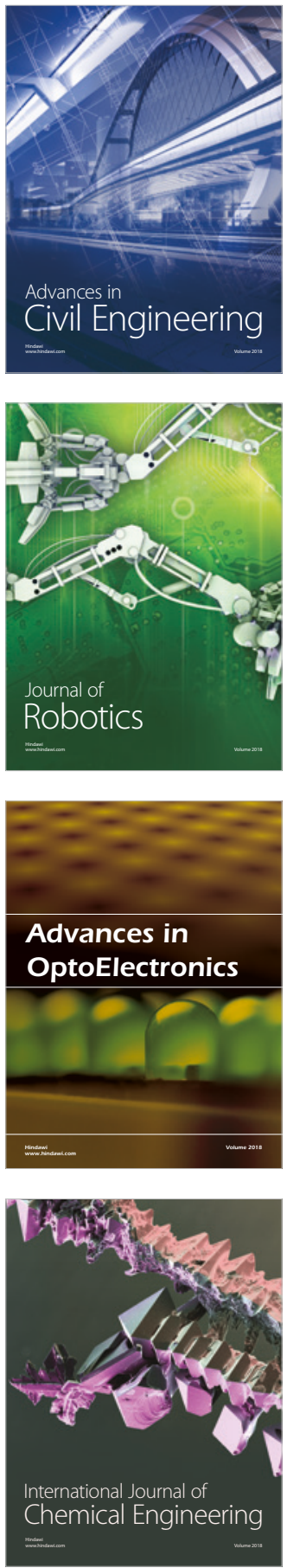

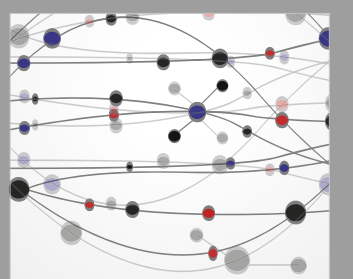

\section{Rotating \\ Machinery}

The Scientific World Journal

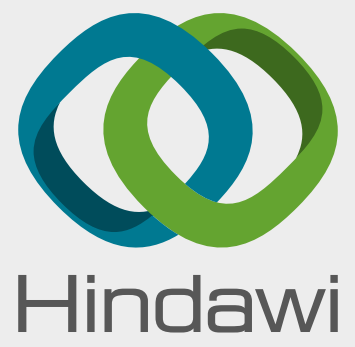

Submit your manuscripts at

www.hindawi.com
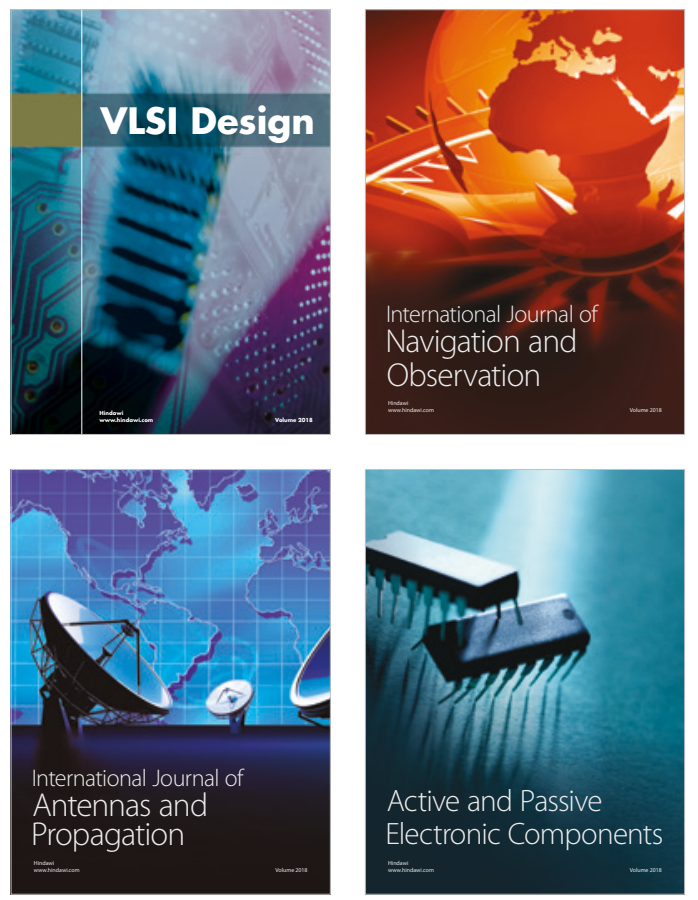
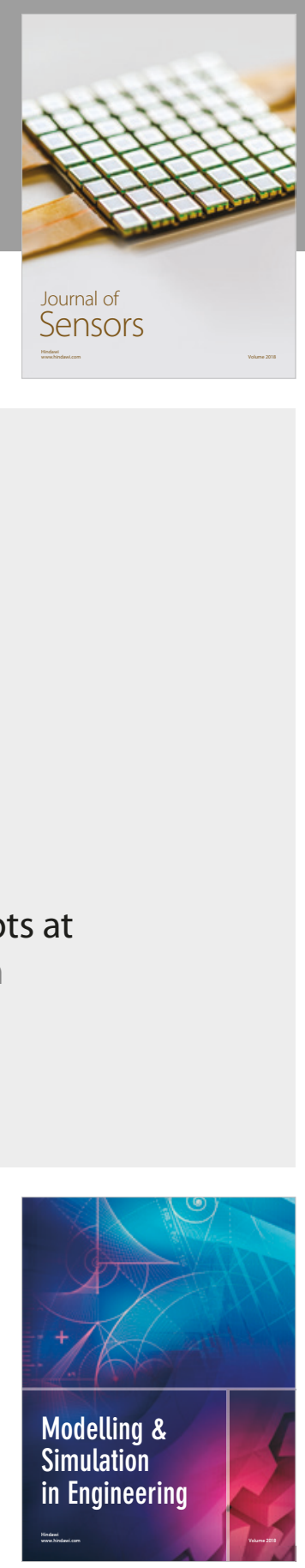

\section{Advances \\ Multimedia}
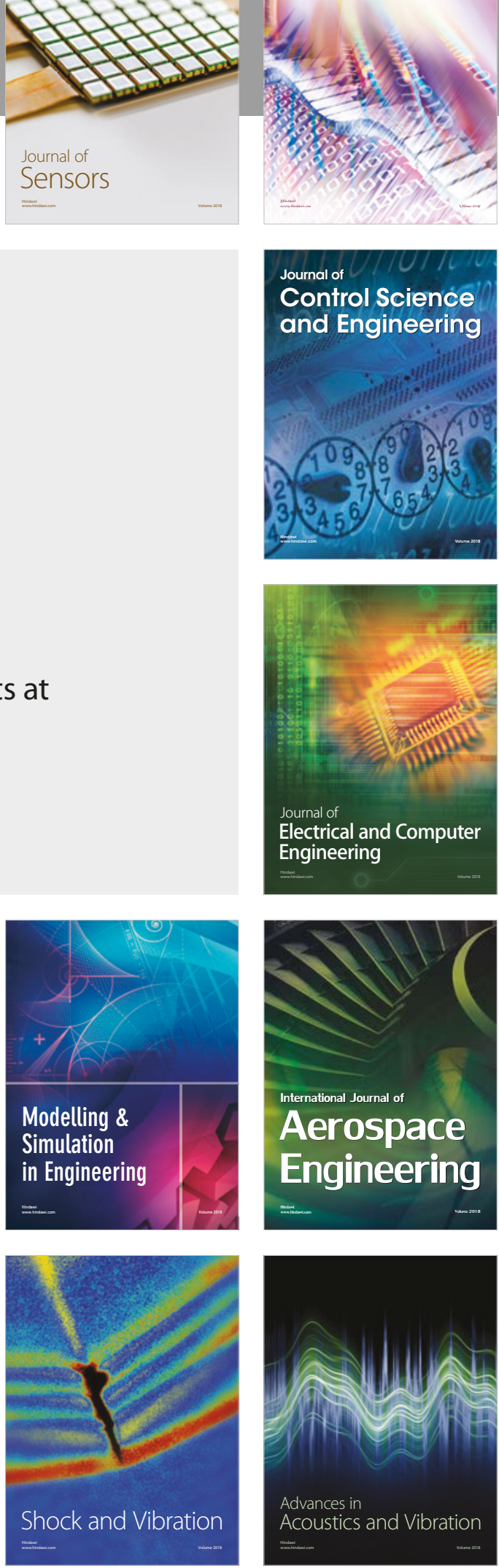